\title{
On Equitorsion Geodesic Mappings of General Affine Connection Spaces
}

\author{
Mića S. Stanković - Svetislav M. Minčić - Ljubica S. Velimirović \\ Milan LJ. Zlatanović
}

ABSTRACT - In the papers [19], [20] several Ricci type identities are obtained by using non-symmetric affine connection. In these identities appear 12 curvature tensors, 5 of which being independent [21], while the rest can be expressed as linear combinations of the others.

In the general case of a geodesic mapping $f$ of two non-symmetric affine connection spaces $G A_{N}$ and $G \bar{A}_{N}$ it is impossible to obtain a generalization of the Weyl projective curvature tensor. In the present paper we study the case when $G A_{N}$ and $G \bar{A}_{N}$ have the same torsion in corresponding points. Such a mapping we name "equitorsion mapping".

With respect to each of mentioned above curvature tensors we have obtained quantities $\mathcal{E}_{\theta}^{i}{ }_{j m n}(\theta=1, \cdots, 5)$, that are generalizations of the Weyl tensor, i.e. they are invariants based on $f$. Among $\underset{\theta}{\mathcal{E}}$ only $\mathcal{E}_{5}$ is a tensor. All these quantities are interesting in constructions of new mathematical and physical structures.

\section{Introduction.}

Geodesic mappings of Riemannian spaces and of affine spaces and their generalizations were investigated by many authors, for examle N. S. Sinjukov [34], J. Mikeš [12]-[18], M. Prvanović [25]-[28, 29], S. Minčić [23], [24], [38]-[41], M. Stanković [35]-[42] and many others.

Consider two $N$-dimensional differentiable manifolds $G A_{N}$ and $G \bar{A}_{N}$ and differentiable mapping $f: G A_{N} \rightarrow G \bar{A}_{N}$. We can consider these manifolds in the common by this mapping system of local coordinates.

Indirizzo degli A.: Faculty of Sciences and Mathematics, University of Niš, Višegradska 33, 18000 Niš, Serbia

E-mails: stmica@ptt.rs, svetislavmincic@yahoo.com, vljubica@pmf.ni.ac.rs, zlatmilan@yahoo.com

AMS Subj. Class.: 53B05. 
Namely, if $f: M \in G A_{N} \rightarrow \bar{M} \in G \bar{A}_{N}$ and if $(\mathcal{U}, \varphi)$ is local chart around the point $M$ it will be $\varphi(M)=x=\left(x^{1}, \cdots, x^{N}\right) \in E^{N}$ (Euclidean $N$-space). In this case, we define for the coordinate mapping in the $G \bar{A}_{N}$ the mapping $\bar{\varphi}=\varphi \circ f^{-1}$, and then

$$
\bar{\varphi}(\bar{M})=\left(\varphi \circ f^{-1}\right)(f(M))=\varphi(M)=x=\left(x^{1}, \cdots, x^{N}\right) \in E^{N},
$$

that is the points $M$ and $\bar{M}=f(M)$ have the same local coordinates. If connection coefficients $L_{j k}^{i}(x)$ and $\bar{L}_{j k}^{i}(x)$, for the connection introduced in $G A_{N}$ and $G \bar{A}_{N}$ respectively, are non-symmetric in lower indices, we call $G A_{N}$ and $G \bar{A}_{N}$ general affine connection spaces.

Although the notion of non-symmetric affine connection is used in several works before A. Einstein, for example in [2] (Eisenhart, 1927), [8] (Hayden, 1932), the use of non-symmetric connection became especially actual after appearance the works of Einstein, relating to create the Unified Field Theory (UFT).

Einstein was not satisfied with his General Theory of Relativity (GTR, 1916), and from 1923. to the end of his life (1955), he worked on various variants of UFT. This theory had the aim to unite the gravitation theory, to which is related GTR, and the theory of electromagnetism.

One says that reciprocal one valued mapping $f: G A_{N} \rightarrow G \bar{A}_{N}$ is geodesic, [23], [24] if geodesics of the space $G A_{N}$ pass to geodesics of the space $G \bar{A}_{N}$. In the corresponding points $M(x)$ and $\bar{M}(x)$ we can put

$$
\bar{L}_{j k}^{i}(x)=L_{j k}^{i}(x)+P_{j k}^{i}(x), \quad(i, j, k=1, \ldots, N),
$$

where $P_{j k}^{i}(x)$ is the deformation tensor of the connection $L$ of $G A_{N}$ according to the mapping $f: G A_{N} \rightarrow G \bar{A}_{N}$.

A necessary and sufficient condition that the mapping $f$ be geodesic [23] is that the deformation tensor $P_{j k}^{i}$ from (2) has the form

$$
P_{j k}^{i}(x)=\delta_{(j}^{i} \psi_{k)}(x)+\xi_{j k}^{i}(x),
$$

where

$$
\begin{gathered}
\psi_{i}=\frac{1}{N+1} P_{\underline{i p}}^{p}=\frac{1}{N+1}\left(\bar{L}_{\underline{i p}}^{p}-L_{\underline{i p}}^{p}\right), \\
\xi_{j k}^{i}=\underset{\substack{j k \\
V}}{i} \frac{1}{2}\left(P_{j k}^{i}-P_{k j}^{i}\right)=\frac{1}{2} P_{[j k]}^{i}
\end{gathered}
$$

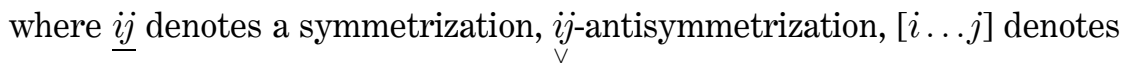
an antisymmetrization without division with respect to the indices $i, j$, and 
also $(i \ldots j)$ denotes a symmetrization without division with respect to indices $i, j$.

In $G A_{N}$ one can define four kinds of covariant derivatives [19, 20]. For example, for a tensor $a_{j}^{i}$ in $G A_{N}$ we have

$$
\begin{aligned}
& a_{j \mid m}^{i}=a_{j, m}^{i}+L_{p m}^{i} a_{j}^{p}-L_{j m}^{p} a_{p}^{i}, \quad a_{j \mid m}^{i}=a_{j, m}^{i}+L_{m p}^{i} a_{j}^{p}-L_{m j}^{p} a_{p}^{i}, \\
& a_{j \mid m}^{i}=a_{j, m}^{i}+L_{p m}^{i} a_{j}^{p}-L_{m j}^{p} a_{p}^{i}, \quad a_{j \mid m}^{i}=a_{j, m}^{i}+L_{m p}^{i} a_{j}^{p}-L_{j m}^{p} a_{p}^{i} .
\end{aligned}
$$

Denote by $|$,$| a covariant derivative of the kind \theta$ in $G A_{N}$ and $G \bar{A}_{N}$ respectively.

While at the Riemannian space (the space of GTR) the connection coefficients are expressed by virtue of symmetric basic tensor $g_{i j}$, at Einstein's works from UFT (1950-1955) the connection between these magnitudes is determined by equations

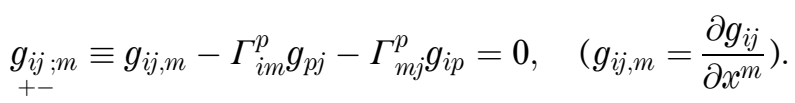

The equation (6) signifies that the index $i$ one treats in the sense of the first kind of derivative (|), and $j$ in the sense of the second one (|).

Proceeding at that sense, Einstein in [1], 1950, for covariant curvature tensor in his theory obtains a Bianchi-type identity:

$$
R_{i k l m ; n}+R_{-+k m n ; l}+R_{i k n l ; m}=0,
$$

where $R_{i k l m}=g_{s i} R_{k l m}^{s}$.

In the case of the space $G A_{N}$ we have five independent curvature tensors [21] (in [21] $R$ is denoted by $\tilde{R}$ ):

$$
\begin{aligned}
& { }_{1}^{R_{j m n}^{i}}=L_{j[m, n]}^{i}+L_{j[m}^{p} L_{p n]}^{i}, \quad{ }_{2}^{R_{j m n}^{i}}{ }^{2}=L_{[m j, n]}^{i}+L_{[m j}^{p} L_{n] p}^{i}, \\
& R_{3 m n}^{i}=L_{j m, n}^{i}-L_{n j, m}^{i}+L_{j m}^{p} L_{n p}^{i}-L_{n j}^{p} L_{p m}^{i}+L_{n m}^{p} L_{[p j]}^{i} \text {, } \\
& \text { (8) } \quad R_{4 m n}^{i}=L_{j m, n}^{i}-L_{n j, m}^{i}+L_{j m}^{p} L_{n p}^{i}-L_{n j}^{p} L_{p m}^{i}+L_{m n}^{p} L_{[p j]}^{i} \text {, } \\
& R_{5}^{i}{ }_{j m n}=\frac{1}{2}\left(L_{j[m, n]}^{i}+L_{[m j, n]}^{i}+L_{j m}^{p} L_{p n}^{i}+L_{m j}^{p} L_{n p}^{i}-L_{j n}^{p} L_{m p}^{i}-L_{n j}^{p} L_{p m}^{i}\right) .
\end{aligned}
$$

By virtue of the geodesic mapping $f: G A_{N} \rightarrow G \bar{A}_{N}$ we obtain tensors $\bar{R}_{\theta}^{i}$, $(\theta=1, \ldots, 5)$, where for example

$$
\bar{R}_{j}^{i} i m n=\bar{L}_{j[m, n]}^{i}+\bar{L}_{j[m}^{p} \bar{L}_{p n]}^{i} .
$$


U. P. Singh ([31], 1968) for a special nonsymmetric connection of M. Prvanović ([27], 1959) uses 2 kinds of covariant derivatives and obtains 3 curvature tensors by forming Ricci identities for a vector.

F. Graif [4] gives geometric interpretations of two kinds parallel displacement, based on non-symmetric connection. M. Prvanović [26], using non-symmetry of the connection, obtains geometric interpretations of of the tensors $R, \ldots, R$, given in (8), and $\mathrm{S}$. Minčić in [22] gives also corresponding interpretations of $R$ from (8).

An application of more kinds of covariant derivative at $L_{N}$ makes possible to express more concise some results. For example, let us consider infinitesimal deformations, defined by

$$
\bar{x}^{i}=x^{i}+\varepsilon z^{i}(x), \quad x=\left(x^{1}, \ldots, x^{N}\right), \quad i=1, \ldots, N,
$$

where $\varepsilon$ is an infinitesimal parameter, $z^{i}(x)$ a vector field. As it is known, a deformed geometric object $\overline{\mathcal{A}}(x)$ (e. g. a tensor, a connection) of the object $\mathcal{A}(x)$ is

$$
\overline{\mathcal{A}}=\mathcal{A}+\varepsilon \mathcal{L}_{z} \mathcal{A},
$$

where $\mathcal{L}_{z} \mathcal{A}$ is the Lie derivative of $\mathcal{A}$ in the direction of the field $z^{i}(x)$. Then, e. g. for a tensor $t_{k l}^{i j}$ ([44] (K. Yano, 1949), [45](K. Yano, 1957) we have:

$$
\mathcal{L}_{z} t_{k l}^{i j}=t_{k l, p}^{i j} z^{p}-z_{, p}^{i} t_{k l}^{p j}-z_{, p}^{j} t_{k l}^{i p}+z_{, k}^{p} t_{p l}^{i j}+z_{, l}^{p} t_{k p}^{i j},
$$

Using the covariant derivatives of one kind, for example the first, instead of partial derivatives, we have

$$
\begin{aligned}
& \text { (13) } \mathcal{L}_{z} t_{k l}^{i j}=t_{k l \mid p}^{i j} z^{p}-z_{1}^{i} t_{k l}^{p j}-z_{\mid p}^{j} t_{k l}^{i p}+ \\
& +z_{\mid k}^{p} t_{p l}^{i j}+z_{\mid l}^{p} t_{k p}^{i j}+2\left(L_{\vee p s}^{i} t_{k l}^{s j}+L_{\vee}^{j} t_{k l}^{i s}+L_{\vee}^{s} t_{s l}^{i j}+L_{\vee p}^{s} t_{k s}^{i j}\right) z^{p},
\end{aligned}
$$

from where we see that Lie derivative is a tensor. But, using more kinds of covariant derivative, we obtain in the considered case [43] (Lj. Velimirović, S. Minčić, M. Stanković):

$$
\mathcal{L}_{z} t_{k l}^{i j}=t_{k l \mid p}^{i j} z^{p}-z_{\mid p}^{i} t_{k l}^{p j}-z_{\mu}^{j} t_{k l}^{i p}+z_{\mid k}^{p} t_{p l}^{i j}+z_{v}^{p} t_{k p}^{i j},
$$

where $(\lambda, \mu, v) \in\{(1,2,2),(2,1,1),(3,4,3),(4,3,4)\}$ i.e. in (14) we have 4 manners of the presenting of Lie derivative, that are more concise than in (13). 
In the case of geodesic mapping $f: A_{N} \rightarrow \bar{A}_{N}$ of the symmetric affine connection spaces $A_{N}$ and $\bar{A}_{N}$ we have an invariant geometric object (Weyl projective tensor)

(15) $W_{j m n}^{i}=R_{j m n}^{i}+\frac{1}{N+1} \delta_{j}^{i} R_{[m n]}+\frac{N}{N^{2}-1} \delta_{[m}^{i} R_{j n]}+\frac{1}{N^{2}-1} \delta_{[m}^{i} R_{n] j}$,

where $R_{j m n}^{i}$ is Riemann-Cristoffel's curvature tensor of the space $A_{N}$, and $R_{j m}$ is Ricci tensor.

The object $W_{j m n}^{i}$ is a tensor and it is called Weyl tensor, or a tensor of projective curvature [34]. Having a geodesic mapping of two general affine connection spaces, we can not find a generalization of Weyl tensor as an invariant of geodesic mapping in general case. For that reason we define a special geodesic mapping.

A mapping $f: G A_{N} \rightarrow G \bar{A}_{N}$ is equitorsion geodesic mapping if the torsion tensor of the spaces $G A_{N}$ and $G \bar{A}_{N}$ are equal. Then from (2), (3) and (5)

$$
\bar{L}_{i j}^{h}-L_{i j}^{h}=\xi_{i j}^{h}(x)=0,
$$

where $i j$ denotes an antisymmetrization with respect to $i, j$.

\section{ET-projective parameter of the first kind.}

Using (3), (9) and (16) we get a relation between the first kind of curvature tensors (8) of the spaces $G A_{N}$ and $G \bar{A}_{N}$

$$
\bar{R}_{1}^{i} i m=\underset{1}{R_{j m n}^{i}}+\delta_{j}^{i} \psi_{[m n]}+\delta_{[m}^{i} \psi_{j} \psi_{j n]}+2 \underset{\vee}{i} L_{\vee n} \psi_{j}+2 L_{v_{n}}^{p} \psi_{p} \delta_{j}^{i},
$$

where we denote

$$
\underset{\theta}{\psi_{m n}}=\underset{\theta}{\psi_{m \mid n}}-\psi_{m} \psi_{n}, \quad \theta=1,2 .
$$

Contracting with respect to $i$ and $n$, from (2) one gets

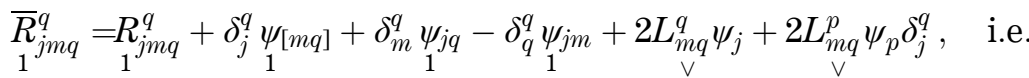

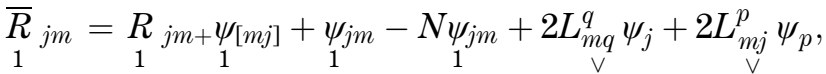

from where

$$
\bar{R}_{j m}=\underset{1}{R_{j m}}-\psi_{1}[j m]+(1-N) \psi_{j}+2 L_{\vee q}^{q} \psi_{j}+2 L_{\vee j}^{p} \psi_{p} .
$$


Here $\bar{R}_{j m}$ and $R_{j m}$ are the first kind Ricci tensors of the spaces $G \bar{A}_{N}$ and $G A_{N}$ respectively. From (18) we obtain

$$
\bar{R}_{[j m]}=\underset{1}{R_{[j m]}}-\underset{1}{2} \psi_{[j m]}+(1-N) \psi_{1}[j m]+\underset{\vee}{q}+\underset{\vee}{q} \psi_{j]}+4 L_{m j}^{p} \psi_{p} .
$$

From (19) and (4) one obtains

(20) $\bar{R}_{1}^{[j m]}=R_{1} R_{[j m]}-(N+1) \psi_{[j[m]}+\frac{2}{N+1} L_{m q}^{q}\left(\bar{L}_{j \underline{p}}^{p}-L_{j \underline{p}}^{p}\right)$

$$
-\frac{2}{N+1} L_{j q}^{q}\left(\bar{L}_{\underline{m p}}^{p}-L_{\underline{m p}}^{p}\right)+\frac{4}{N+1} L_{\underline{v}}^{p}\left(\bar{L}_{\underline{p q}}^{q}-L_{\underline{p q}}^{q}\right)
$$

from where

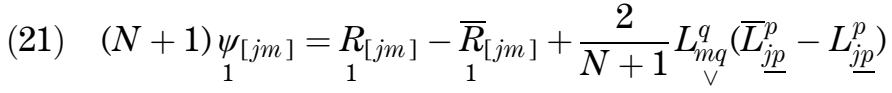

$$
\begin{aligned}
& -\frac{2}{N+1} L_{j q}^{q}\left(\bar{L}_{\underline{m p}}^{p}-L_{\underline{m p}}^{p}\right)+\frac{4}{N+1} L_{\underset{v}{p}}^{p}\left(\bar{L}_{\underline{p q}}^{q}-L_{\underline{p q}}^{q}\right) .
\end{aligned}
$$

Because of (18), (4) and (21), we have

$$
\begin{aligned}
& \bar{R}_{j m}=R_{1}-\frac{1}{N+1}\left[R_{1}[j m]-\bar{R}_{[j m]}+\frac{2}{N+1} L_{m q}^{q}\left(\bar{L}_{j \underline{p}}^{p}-L_{j \underline{p}}^{p}\right)\right. \\
& \left.-\frac{2}{N+1} L_{j q}^{q}\left(\bar{L}_{\underline{m p}}^{p}-L_{\underline{m p}}^{p}\right)+\frac{4}{N+1} L_{m j}^{p}\left(\bar{L}_{\underline{p q}}^{q}-L_{\underline{p q}}^{q}\right)\right]
\end{aligned}
$$

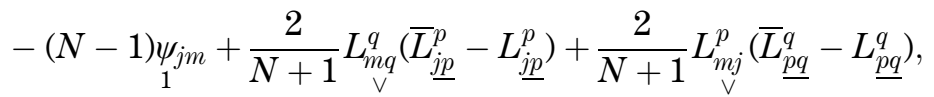

that is

$$
\begin{aligned}
& (N-1) \psi_{1}=R_{1}-\bar{R}_{j m}-\frac{1}{N+1}\left[R_{1}[j m]-\bar{R}_{1}{ }_{1 j m]}+\frac{2}{N+1} L_{m q}^{q}\left(\bar{L}_{\underline{j p}}^{p}-L_{\underline{j p}}^{p}\right)\right. \\
& \left.-\frac{2}{N+1} L_{j q}^{q}\left(\bar{L}_{\underline{m p}}^{p}-L_{\underline{m p}}^{p}\right)+\frac{4}{N+1} L_{m j}^{p}\left(\bar{L}_{\underline{p q}}^{q}-L_{\underline{p q}}^{q}\right)\right]
\end{aligned}
$$

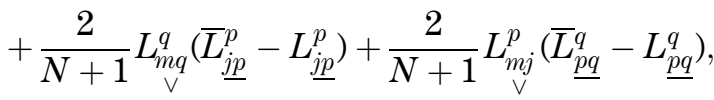

from where

$$
\begin{aligned}
& \left(N^{2}-1\right) \psi_{j}=\left(N R_{j m}+\underset{1}{R_{m j}}\right)-\left(N \bar{R}_{j m}+\bar{R}_{m j}\right)+\frac{2 N}{N+1} L_{m q}^{q}\left(\bar{L}_{j \underline{p}}^{p}-L_{j \underline{p}}^{p}\right) \\
& +\frac{2}{N+1} L_{j q}^{q}\left(\bar{L}_{\underline{m p}}^{p}-L_{\underline{m p}}^{p}\right)+2 L_{\underline{\sim}}^{p}\left(\bar{L}_{\underline{p q}}^{q}-L_{\underline{p q}}^{q}\right) \frac{N-1}{N+1} .
\end{aligned}
$$


Substituting (22) at (2), by help of (16), it is easy to prove that

$$
\overline{\mathcal{E}}_{1}^{i}{ }_{1 m n}=\mathcal{E}_{1}^{i},
$$

where we have denoted

$$
\begin{aligned}
& \mathcal{E}_{1}^{i}{ }_{j m n}=R_{1}^{i} i m n+\frac{N}{N+1} \delta_{j}^{i} R_{[m n]}+\frac{N}{N^{2}-1} \delta_{[m}^{i} R_{j n]}+\frac{1}{N^{2}-1} \delta_{[m}^{i} \underset{1}{R_{n] j}} \\
& +\frac{2}{(N+1)^{2}(N-1)} L_{\underline{[m p}}^{p} \delta_{n]}^{i} L_{j q}^{q}-\frac{2}{(N+1)^{2}} L_{\underline{[m p}}^{p} \delta_{j}^{i} L_{n] q}^{q} \\
& +\frac{2 N}{(N+1)\left(N^{2}-1\right)} L_{j \underline{p}}^{p} \delta_{[n}^{i} L_{m] q}^{q}
\end{aligned}
$$

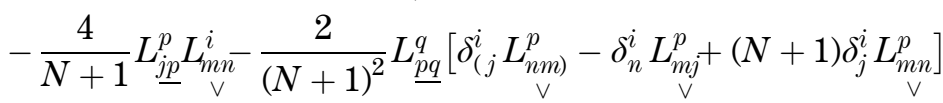

Obviously, the magnitude $\mathcal{E}_{1 \text { jmn }}^{i}$ is not a tensor and we call it the $E T$-projective parameter of the first kind for the mapping $f: G A_{N} \rightarrow G \bar{A}_{N}$. So, we have

THEOREM 1. The ET-projective parameter of the first kind $\mathcal{E}_{1}^{i}$ (24) is an invariant of the equitorsion mapping $f: G A_{N} \rightarrow G \bar{A}_{N}$.

\section{ET-projective parameter of the second kind.}

For the second kind of curvature tensors $R$ and $\bar{R}$ (8) of the spaces $G A_{N}$ and $G \bar{A}_{N}$ respectively, we get the relation

$$
\bar{R}_{2}^{i} i m=R_{2}^{i} i m n+\delta_{j}^{i} \psi_{[m n]}+\delta_{[m}^{i} \psi_{2}^{i n]}+2 L_{\vee m}^{i} \psi_{j}+2 \underset{\vee}{L_{n m}^{p}} \psi_{p} \delta_{j}^{i},
$$

Analogously to previous case, we get

$$
\overline{\mathcal{E}}_{2}^{i}{ }_{j m n}=\mathcal{E}_{2}^{i} \text { jmn }
$$

where is

$$
\begin{aligned}
& \mathcal{E}_{2}^{i}{ }_{j m n}=R_{2}^{i} i m n+\frac{N}{N+1} \delta_{j}^{i} R_{1 m n]}+\frac{N}{N^{2}-1} \delta_{[m}^{i} \underset{2}{i} R_{j n]}+\frac{1}{N^{2}-1} \delta_{[m}^{i} R_{2} R_{n] j} \\
& +\frac{2}{(N+1)^{2}(N-1)} L_{\underline{[m p}}^{p} \delta_{n]}^{i} L_{q j}^{q}-\frac{2}{(N+1)^{2}} L_{\underline{[m p}}^{p} \delta_{j}^{i} L_{\substack{q n] \\
\vee}}^{q} \\
& +\frac{2 N}{(N+1)\left(N^{2}-1\right)} L_{j p}^{p} \delta_{[n}^{i} L_{q m]}^{q} \\
& -\frac{4}{N+1} L_{\underline{j p}}^{p} L_{\vee}^{i}-\frac{2}{(N+1)^{2}} L_{\underline{p q}}^{q}\left[\delta_{j}^{i} L_{\underset{v n}{p}+}^{p} \delta_{(m}^{i} L_{\substack{j n \\
\vee}}^{p}+(N+1) \delta_{j}^{i} L_{\mathfrak{v}}^{p}\right]
\end{aligned}
$$


The magnitude $\mathcal{E}_{2}^{i}{ }_{j m n}$ is not a tensor and we call it ET-projective parameter of the second kind. In this case we have

THEOREM 2. The ET-projective parameter of the second kind $\mathcal{E}_{2}^{i}{ }_{j m n}(27)$ is an invariant of the equitorsion mapping $f: G A_{N} \rightarrow G \bar{A}_{N}$.

\section{ET-projective parameter of the third kind.}

In the case of the third kind of curvature tensors $R$ and $\bar{R}$ (8) of the spaces $G A_{N}$ and $G \bar{A}_{N}$ we get

$$
\bar{R}_{3}^{i} i_{3 m n}=\underset{3}{R_{j m n}}+\delta_{(j}^{i} \psi_{m) n}-\delta_{(j}^{i} \psi_{n) m}+2 \psi_{(n} L_{m \vee j}^{i}
$$

Since

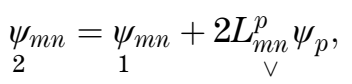

from (28) one obtains

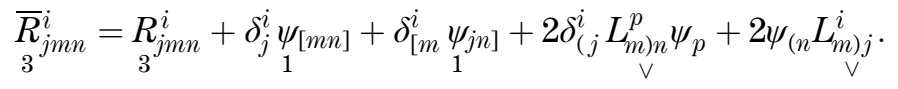

Contracting in (29) over $i, n$, we obtain

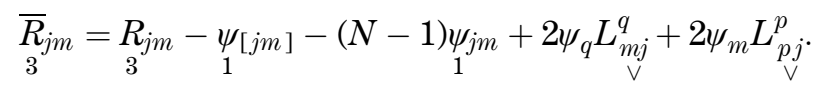

Alternating the last equation with respect to $j, m$, we have

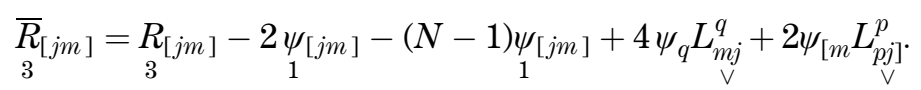

By using (4), we get

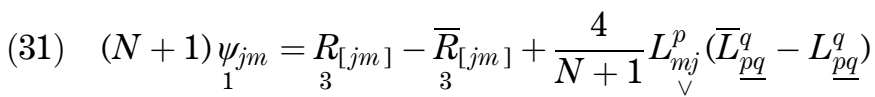

$$
\begin{aligned}
& +\frac{2}{N+1} L_{p j}^{p}\left(\bar{L}_{\underline{m q}}^{q}-L_{\underline{m q}}^{q}-\frac{2}{N+1} L_{p m}^{p}\left(\bar{L}_{\underline{j q}}^{q}-L_{\underline{j q}}^{q}\right),\right.
\end{aligned}
$$


By virtue of (4), (31) and (30) we obtain

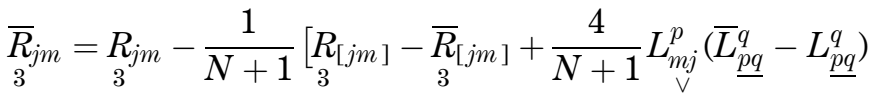

$$
\begin{aligned}
& +\frac{2}{N+1} L_{\underline{p j}}^{p}\left(\bar{L}_{\underline{m q}}^{q}-L_{\underline{m q}}^{q}\right)-\frac{2}{N+1} L_{p m}^{p}\left(\bar{L}_{\underline{j q}}^{q}-L_{\underline{j q}}^{q}\right]-(N-1) \psi_{1}{ }_{j m} \\
& +\frac{2}{N+1} L_{\underline{m j}}^{p}\left(\bar{L}_{\underline{p q}}^{q}-L_{\underline{p q}}^{q}\right)+\frac{2}{N+1} L_{p j}^{p}\left(\bar{L}_{\underline{m q}}^{q}-L_{\underline{m q}}^{q}\right),
\end{aligned}
$$

i.e.

$$
\begin{aligned}
& \left(N^{2}-1\right) \psi_{1}=\left(N_{3} R_{j m}+R_{3}\right)-\left(N \bar{R}_{3 m}+\bar{R}_{3 j}\right)+2 L_{m j}^{p}\left(\bar{L}_{\underline{p} q}^{q}-L_{\underline{p q}}^{q}\right) \frac{N-1}{N+1} \\
& +2 L_{\underline{p}}^{p}\left(\bar{L}_{\underline{m q}}^{q}-L_{\underline{m q}}^{q}\right) \frac{N}{N+1}+\frac{2}{N+1} L_{p m}^{p}\left(\bar{L}_{j \underline{q}}^{q}-L_{\underline{j q}}^{q}\right) .
\end{aligned}
$$

Substituting (32) into (29) using the condition (4) we obtain

$$
\overline{\mathcal{E}}_{3}^{i}{ }_{j m n}=\mathcal{E}_{3}^{i} \text { jmn }
$$

where

$$
\begin{aligned}
& \mathcal{E}_{3}^{i}{ }_{j m n}=R_{3}^{i} i m n+\frac{1}{N+1} \delta_{j}^{i} R_{[m n]}+\frac{N}{N^{2}-1} \delta_{[m}^{i} R_{3} R_{j n]}+\frac{1}{N^{2}-1} \delta_{[m}^{i} R_{n] j} \\
& +\frac{2}{(N-1)(N+1)^{2}} L_{\underline{j q}}^{q} \delta_{[n}^{i} L_{p m]}^{p}+\frac{2}{(N+1)^{2}} L_{\underline{[m q}}^{q} \delta_{j}^{i} L_{p n]}^{p} \\
& +\frac{2 N}{(N+1)^{2}(N-1)} L_{\underline{[m q}}^{q} \delta_{n]}^{i} L_{\underset{\vee}{p}}^{p}-\frac{2}{(N+1)} L_{\underline{(m q}}^{q} L_{n j}^{i}
\end{aligned}
$$

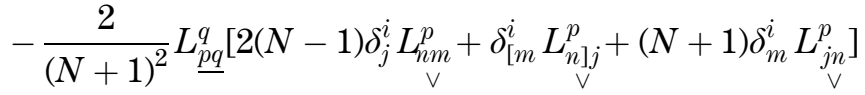

The magnitude $\mathcal{E}_{3}^{i}$ imn is not a tensor and we call it ET-projective parameter of the third kind. In this case we have

THEOREM 3. The ET-projective parameter of the third kind $\mathcal{E}_{3}^{i}$ jmn $(34)$ is an invariant of the equitorsion mapping $f: G A_{N} \rightarrow G \bar{A}_{N}$.

\section{ET-projective parameter of the fourth kind.}

For the curvature tensors of the fourth kind $R_{4}$ and $\bar{R}(8)$ of the space $G A_{N}$ and $G \bar{A}_{N}$ respectively, we get

$$
\bar{R}_{4}^{i} i m=R_{4}^{i} i m n+\delta_{(j}^{i} \psi_{m) n}-\delta_{(j}^{i} \psi_{n) m}+2 \psi_{(m} L_{n) j}^{i},
$$


from where

$$
\overline{\mathcal{E}}_{4 m n}^{i}=\mathcal{E}_{4}^{i}{ }_{j m n},
$$

where

$$
\begin{aligned}
& \left.\mathcal{E}_{4}^{i}{ }_{j m n}=R_{4}^{i} i m n+\frac{1}{N+1} \delta_{j}^{i} R_{4}[m n]+\frac{N}{N^{2}-1} \delta_{[m}^{i} \underset{4}{i} R_{j n}\right]+\frac{1}{N^{2}-1} \delta_{[m}^{i} \underset{4}{i} R_{n] j} \\
& +\frac{2}{(N-1)(N+1)^{2}} L_{j \underline{q}}^{q} \delta_{[n}^{i} L_{p m]}^{p}-\frac{2}{(N+1)^{2}} L_{\underline{(m q}}^{q} \delta_{j}^{i} L_{p n)}^{p} \\
& +\frac{2 N}{(N+1)^{2}(N-1)} L_{\underline{(m q}}^{q} \delta_{n)}^{i} L_{p j}^{p}-\frac{2}{(N+1)} L_{\underline{\underline{[m q}}}^{q} L_{n] j}^{i}
\end{aligned}
$$

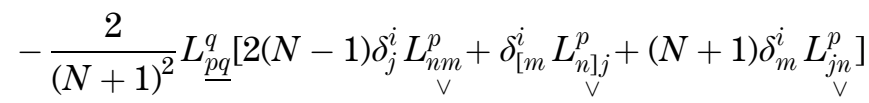

The magnitude $\mathcal{E}_{j m n}^{i}$ is not a tensor and we call it ET-projective parameter of the fourth kind. In this case we have

THEOREM 4. The ET-projective parameter of the fourth kind $\mathcal{E}_{4}^{i}$ (37) is an invariant of the equitorsion mapping $f: G A_{N} \rightarrow G \bar{A}_{N}$.

\section{ET-projective curvature tensor.}

For the curvature tensors $R$ and $\bar{R}$ (8) of the space $G A_{N}$ and $G \bar{A}_{N}$ respectively, we find the relation

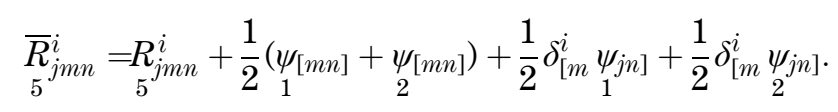

Denoting

$$
\underset{12}{\psi_{j n}}=\frac{1}{2}\left(\underset{1}{\psi_{j n}}+\underset{2}{\psi_{j n}}\right)
$$

we can write (38) in the form

$$
\bar{R}_{5}^{i}{ }_{j m n}=R_{5}^{i} i m n+\delta_{j 2}^{i} \psi_{[m n]}+\delta_{[m}^{i} \psi_{12} \psi_{j n]} .
$$

Eliminating $\underset{12}{\psi_{m n}}$ from (40) by analogous procedure as in the previous cases, one obtains

$$
\overline{\mathcal{E}}_{5}^{i}{ }_{j m n}=\mathcal{E}_{5}^{i}{ }_{j m n},
$$


where

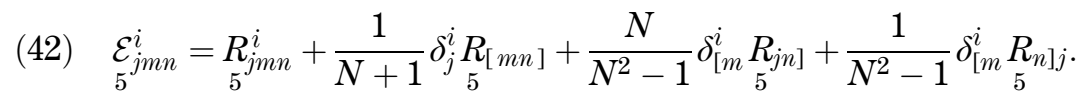

In contrast to the previous cases, when $\mathcal{E}_{\theta}^{i}(\theta=1, \ldots, 4)$ are not tensors, the magnitude $\mathcal{E}_{5}^{i}$ isn a tensor. We call it ET-projective curvature tensor. We see that the following is valid

THEOREM 5. The ET-projective tensor $\mathcal{E}_{5}^{i}$ jmn $(42)$ is an invariant of the equitorsion mapping $f: G A_{N} \rightarrow G \bar{A}_{N}$.

\section{Conclusion.}

In the case of generalized Riemannian space $\left(G R_{N}\right)$ the connection coefficients are defined by means of a non-symmetric basic tensor [3], [19], [20], [32] and they are non-symmetric too. ET-projective parameters $E_{\theta}^{i}{ }_{j m n}$ and the projective tensor $E_{5}^{i}$, obtained as invariants of a map $f: G R_{N} \rightarrow G \bar{R}_{N}$ are particular cases of obtained here parameters $\mathcal{E}_{\theta}^{i}{ }_{j m n} \quad(\theta=1, \cdots 4)$ and of the tensor $\mathcal{E}_{5}^{i}$.mn . For example,

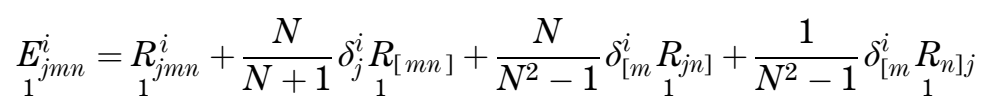

$$
\begin{aligned}
& -\frac{4}{N+1} L_{\underline{j p}}^{p} L_{\underset{\vee}{m}}^{i}-\frac{2}{(N+1)^{2}} L_{\underline{p q}}^{q}\left[\delta_{j}^{i} L_{v_{\vee}}^{p}+\delta_{[m}^{i} L_{\vee]^{j}}^{p}+(N+1) \delta_{j}^{i} L_{\underset{\vee}{p}}^{p}\right]
\end{aligned}
$$

When $G A_{N}\left(G R_{N}\right)$ reduces to the Riemannian space, the magnitudes $\mathcal{E}(E)$, $(\theta=1, \cdots 5)$ reduce to the Weyl tensor [34]

$$
W_{j m n}^{i}=R_{j m n}^{i}+\frac{1}{N-1} \delta_{[m}^{i} R_{j n]}
$$

Acknowledgments. The authors are grateful to the anonymous referees for comments and suggestions, which were helpful in improving the manuscript.

The authors gratefully acknowledge support from the research projects 144032 of the Serbian Ministry of Science. 


\section{REFERENCES}

[1] A. EInstein, The Bianchi identities in the generalized theory of gravitation, Canadian Jour. Math., 2 (1950), pp. 120-128.

[2] L. P. Eisenhart, Non-Riemannian geometry, New York, 1927.

[3] L. P. Eisenhart, Generalized Riemannian spaces I, Nat. Acad. Sci. USA, 37 (1951), pp. 311-315.

[4] F. GRAIF, Sulla possibilità di costruire parallelogrami chiusi in alcune varietà a torsione, Bollet. Un. mat. Italiana, Ser. III, 7 (1952), pp. 132-135.

[5] G. S. HALL - D. P. LONIE, The principle of equivalence and projective structure in spacetimes, Class. Quantum Grav., 24 (2007), pp. 3617-3636.

[6] G. S. HALL - D. P. LONIE, The principle of equivalence and cosmological metrics, J. Math. Phys., 49 (2008), 022502.

[7] G. S. Hall - D. P. Lonie, Projective equivalence of Einstein spaces in general relativity, Class. Quantum Grav., 26 (2009), 125009.

[8] H. A. HAYden, Subspaces of a space with torsion, Proc. London math. soc., 34 (2) (1932), pp. 27-50.

[9] I. Hinterleitner - J. Mikeš, On F-planar mappings of spaces with affine connections, Note di Matematica, 27 n. 1 (2007), pp. 111-118.

[10] I. Hinterleitner - J. Mikeš - J. Stranska, Infinitesimal F-Planar Transformations, Russian Mathematics (Iz. VUZ), Vol. 52, No. 4 (2008), pp. $13-18$.

[11] M. JukL - L. Juklova - J. Mikeš, On generalized trace decompositions Problems, Proceedings of the Third International Conference dedicated to 85-th birthday of Professor Kudrijavcev (2008), pp. 299-314.

[12] J. Mikě̌, On geodesic mappings of Einstein spaces (In Russian), Mat. zametki, 28 (1980), pp. 313-317.

[13] J. Mıkeš, Geodesic mappings of special Riemannian spaces, Coll. Math. Soc. J. Bolyai, 46. Topics in Diff. Geom., Debrecen (Hungary) (1984), pp. 793-813.

[14] J. MIKEŠ, On an order of special transformartion of Riemannian spaces, Dif. Geom. and Apl., Proc. of the Conf. Dubrovnik, 3, (1988), pp. 199-208.

[15] J. Mikeš, Holomorphically projective mappings and their generalizations, Itogi Nauki i Tekhniky, Ser. Probl. Geom. VINITI, 1988.

[16] J. MIkEš, Geodesic mappings of affine-connected and Riemannian spaces, J. Math. Sci. New York, (1996), pp. 311-333.

[17] J. MikeŠ - V. KiosaK - A. Vanžurová, Geodesic Mappings of Manifolds with Affine Connection, Olomounc, 2008.

[18] J. Mikeš - G. A. StaRKo, K-concircular vector fields and holomorphically projective mappings on Kählerian spaces, Rend. del Circolo di Palermo, 46 (1997), pp. 123-127.

[19] S. M. MINčić, Ricci identities in the space of non-symmetric affine connection, Mat. Vesnik, 10 (25) (1973), pp. 161-172.

[20] S. M. MINČÍ́, New commutation formulas in the non-symmetric affine connection space, Publ. Inst. Math. (Beograd) (N. S), 22 (36) (1977), pp. 189-199.

[21] S. M. MiNčIĆ, Independent curvature tensors and pseudotensors of spaces with non-symmetric affine connection, Coll. Math. Soc. János Bolyai, 31 (1979), pp. 45-460. 
On Equitorsion Geodesic Mappings of General Affine Connection Spaces 89

[22] S. M. MinčIć, Geometric interpretations of curvature tensors and pseudotensors of the spaces with non symmetric affine connection, Publ. Inst. Math., 47 (61) (1990), pp. 113-120 (in Russian).

[23] S. M. MinčIĆ - M. S. STANKović, On geodesic mapping of general affine connection spaces and of generalized Riemannian spaces, Mat. vesnik, 49 (1997), pp. 27-33.

[24] S. M. Minčić - M. S. Stanković, Equitorsion geodesic mappings of generalized Riemannian spaces, Publ. Inst. Math. (Beograd) (N.S), 61 (75) (1997), pp. 97-104.

[25] M. Prvanović, Holomorphically projective transformations in a locally product Riemannian spaces, Math. Balkanica, 1 (1971), pp. 195-213.

[26] M. Prvanović, Four curvature tensors of non-symmetric affine connexion (in Russian), Proceedings of the conference "150 years of Lobachevski geometry”, Kazan' 1976, Moscow 1997, pp. 199-205.

[27] M. Prvanović, Relative Frenet formulae for a curve in a subspace of a Riemannian space, Tensor, N. S., 9 (1959), pp. 190-204.

[28] M. Prvanović, A note on holomorphically projective transformations of the Kähler space, Tensor, N. S. Vol. 35 (1981), pp. 99-104.

[29] M. Prvanović, $\pi$-Projective Curvature Tensors, Annales Univ. Maria CurieSklodowska, Lublin - Polonia, XLI, 16 (1986), pp. 123-133.

[30] ZH. RADULOVICH, Holomorphically projective mappings of parabolically Kählerian spaces, Math. Montisnigri, Vol. 8 (1997), pp. 159-184.

[31] U. P. SINGH, On relative curvature tensors in the subspace of a Riemannian space, Rev. de la fac. des sc. Istanbul, 33 (1968), pp. 69-75.

[32] K. D. Singh, On generalized Riemann spaces, Riv. Mat. Univ. Parma, 7 (1956), pp. 125-138.

[33] M. SHIHA, On the theory of holomorphically projective mappings parabolically Kählerian spaces, Differ. Geometry and Its Appl. Proc. Conf. Opava. Silesian Univ., Opava (1993), pp. 157-160.

[34] N. S. Sinyukov, Geodesic mappings of Riemannian spaces (in Rusian), Nauka, Moskow, 1979.

[35] M. S. Stanković, First type almost geodesic mappings of general affine connection spaces, Novi Sad J. Math., 29, No. 3 (1999), pp. 313-323.

[36] M. S. Stanković, On a canonic almost geodesic mappings of the second type of affine spaces, Filomat (Niš), 13 (1999), pp. 105-114.

[37] M. S. Stanković, On a special almost geodesic mappings of the third type of affine spaces, Novi Sad J. Math., 31, No. 2 (2001), pp. 125-135.

[38] M. S. Stanković - S. M. Minčić, New special geodesic mappings of generalized Riemannian space, Publ. Inst. Math. (Beograd) (N.S), 67 (81) (2000), pp. 92-102.

[39] M. S. Stanković - S. M. Minčić, New special geodesic mappings of affine connection spaces, Filomat, 14 (2000), pp. 19-31.

[40] M. S. Stanković - S. M. Minčić - LJ. S. Velimirović, On holomorphically projective mappings of generalized Kählerian spaces, Mat. vesnik, 54 (2002), pp. $195-202$.

[41] M. S. Stanković - S. M. Minčić - LJ. S. Velimirović, On equitorsion holomorphically projective mappings of generalised Kählerian spaces, Czechoslovak Mathematical Journal, 54 (129) (2004), pp. 701-715. 
90 M. S. Stanković - S. M. Minčić - L. S. Velimirovié - M. Lj. Zlatanović

[42] M. S. Stanković - LJ. M. Zlatanović - LJ. S. Velimirović, Equitorsion holomorphically projective mappings of generalized Kählerian space of the first kind, Czechoslovak Mathematical Journal, Vol. 60, No. 3 (2010), pp. 635-653.

[43] L. S. Velimirović - S. M. Minčić - M. S. Stanković, Infinitesimal Deformations and Lie Derivative of a Non-symmetric Affine Connection Space, Acta Univ. Palacki. Olomuc, Fac. rer. nat., Mathematica, 42 (2003), pp. 111-121.

[44] K. Yano, Sur la théorie des déformations infinitesimales, Journal of Fac. of Sci. Univ. of Tokyo, 6 (1949), pp. 1-75.

[45] K. YAno, The theory of Lie derivatives and its applications, N-Holland Publ. Co. Amsterdam, 1957.

Manoscritto pervenuto in redazione il 18 settembre 2009. 